\title{
DYNAMIC INTERACTION OF MOBILE DEVICE AND DATABASE FOR SIMPLIFICATION OF CALL CENTER TASKS
}

\author{
Jagadeesh Prabhakaran $^{1}$, Abraham M.K ${ }^{2}$, G. Gautham ${ }^{3}$, K. Valarmathi $^{4}$ \\ ${ }^{1}$ Student, Department of CSE, Panimalar Engineering College, Chennai, India \\ ${ }^{2}$ Student, Department of CSE, Panimalar Engineering College, Chennai, India \\ ${ }^{3}$ Student, Department of CSE, Panimalar Engineering College, Chennai, India \\ ${ }^{4}$ Professor, Department of CSE, Panimalar Engineering College, Chennai, India
}

\begin{abstract}
In today's world call centers and BPO's (Business Processing and Outsourcing) have become a big business and thousands of people are employed in the voice process to provide assistance to users of various products and services from across the globe. Though the positive aspect of this system is that it has provided a good number of job opportunities, the dark side lies hidden. These employees are subject to a lot of stress attending hundreds of calls in a day and patiently handing each and every caller. On the other hand the caller also gets impatient when his call is on hold or not attended for a long duration. It more often leads to conflicts between the caller and the teller. This can be avoided by the usage of mobile applications to interact with the database, where the system would be predominantly based on textual data and not voice calls. It is carried out using the SOAP protocol and server for implementation purpose.
\end{abstract}

Keywords: Mobile Computing, Database, Network

\section{INTRODUCTION}

Mobile computing is a trend in technology that is growing at a very fast pace in today's world. This can be utilized at the best way possible to solve any issue in technology. In recent times with the development of new platforms in mobile operating systems such as Android, iOS and Windows Mobile, there is a rise in the trend of mobile applications that are developed for a mobile device operating on a specific OS. These applications are used for a variety of purpose from instant messaging to weather forecast and gaming. The employment of an application for reporting to the call centers will solve multiple issues.

\section{PROBLEM DEFINITION}

Consider a mobile user who is unable to use GPRS on his mobile device and wants to report the issue to his service provider by calling on their customer care number. He calls at a busy part of the day, enters the dial according to the IVRS system, waits and waits for so long that he gets impatient by the time his call is attended by the customer care executive and eventually shouts at the teller. On the other hand the executive is at his highest level of stress attending numerous calls over the day and sorting out issues. This system is primarily to sort this problem and propose a solution.

\section{RELATED WORKS}

In order to propose this system a number of related papers have been referred to gain guidance on how applications can be developed and how they can be made to interact with the database server. A complete idea about the client side web service SOAP [1] was taken to be used here. The general factors to be looked at while connecting a database and mobile device was taken from the survey paper of Daniel Barbara [2] proposed in 1999 A couple of papers were studied on how to make a secure applications [3], since security is very vital in this area of application as the data transmitted could be personal. The paper involving data mining from a cloud through a mobile device gave vital clues on data retrieval from a server, though a cloud in specific is not used in the proposed system. The kind of web service that would be required for this system was decided after going through the paper on mobile learning applications [4] using web service.

\section{PROPOSED SYSTEM}

This system involves the knot of a cluster of 2 individual units. The initial one is the mobile application, which depends on the platform of development. The second one is the dynamic database system which interacts with the mobile application directly. The knot between the two components is tied by the network, where comes the work of the protocols and the web service which enhances interaction of the application with the database. The work of the middle man here is that instead of attending the call and answering the 
caller personally he can send a text as a reply which can solve the issue.

\subsection{Mobile Application}

The mobile application can be developed using languages that pertain to the respective platform of usage. We live in an era where a mobile phone or device has become so common and the world's mobile device industry is dominated by the 3 makers. The languages to be used to develop applications are JAVA for Android, Objective C for iOS and C\# of the .NET framework for Windows Mobile. So development of applications in these 3 languages will be more than important. The front end can be designed in any prototyping tool available today.

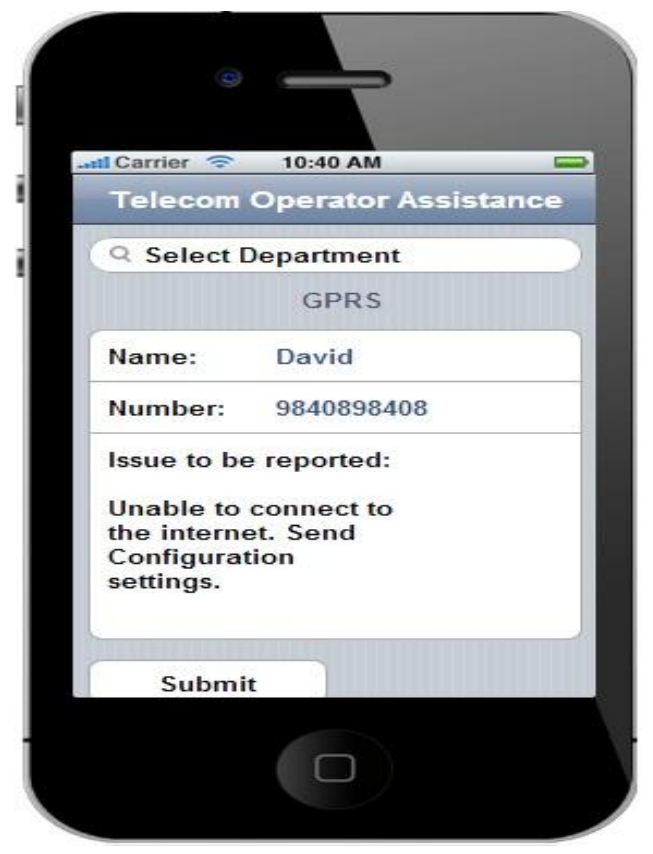

Fig1: Screenshot of Mobile Application

\subsection{Web Application}

The data that is received by the call center executive can be viewed as a Form that holds the data report received from the mobile device. There is developed using Active Server Page in the .NET framework. These forms are simply generated in Visual Basics.

\subsection{Database Server}

The most important component of this system is the database server. The database is designed using MySQL a product of Oracle that can hold records of data as received and segregated in the same pattern as sent by the mobile device. An alternative would be to stick on to the contemporary Microsoft's database system- MSSQL. For the implementation purpose of the system a demonstration can be shown using a basic Apache Tomcat Server Version 6.0. But depending on its use in the respective industry's call center and the load/number of reports it will have to handle in unit time an extensive shared or dedicated server can be deployed.

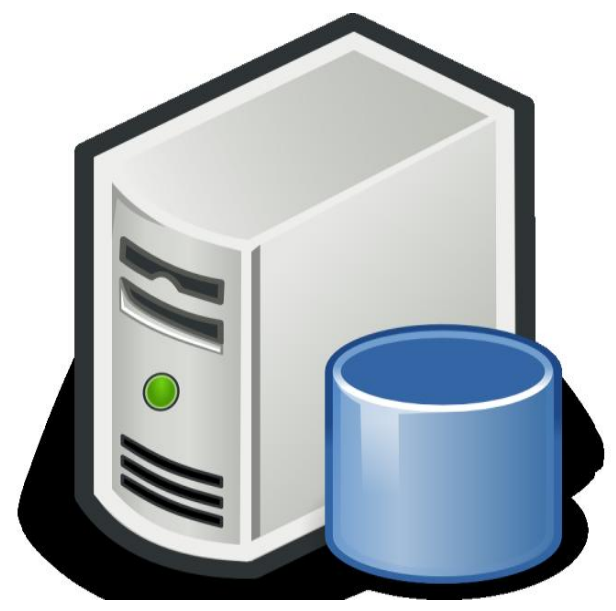

Fig. 2: Database Server

\subsection{Network End}

A protocol SOAP (Simple Object Access Protocol) is deployed here for the exchange of structured information. This works on the Application Layer of the network. It works along with other protocols such as SMTP (Simple Mail Transfer Protocol) and HTTP (Hyper Text Transfer Protocol). The usage of a web service is very essential here to transfer the correct data to and from the database. An SMS gateway is also deployed in the system for sending the reference numbers in the form of auto generated text messages.

\section{SYSTEM ARCHITECTURE}

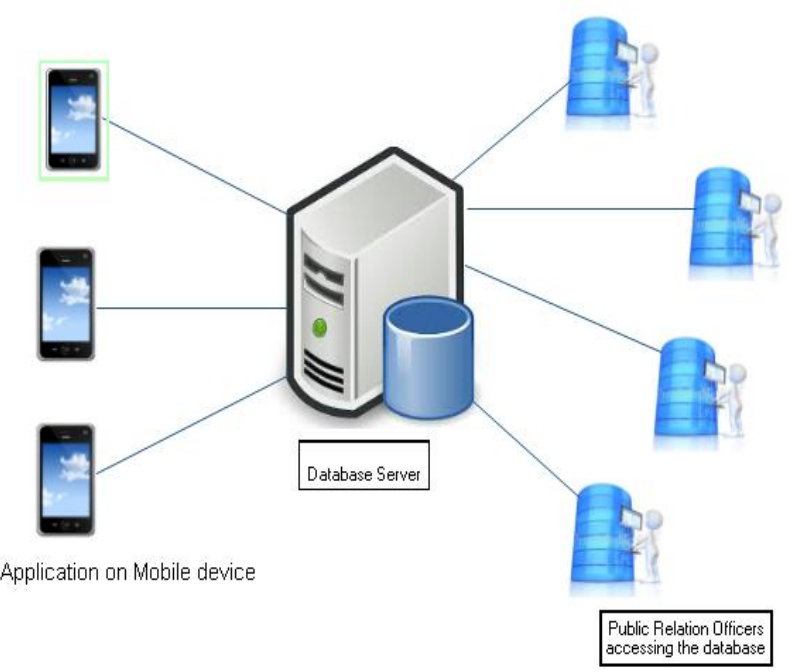

Fig. 3: Architecture Diagram 
In this system the user installs the respective mobile application on his hand held device and types his report on a particular issue and sends it. The message generated uses the GPRS (General Packet Radio Service)/ Wi-Fi/ Mobile Internet facility on his device to travel over the network and reaches the customer service executive as a FORM in his web application. Once the message is received, it gets stored in the systems database and an auto generated reference number is sent to the user using the SMS gateway. When the executive reads the FORM he sends a corresponding reply to the issue reported by the user. This reply is received on the same application and the necessary assistance is provided.

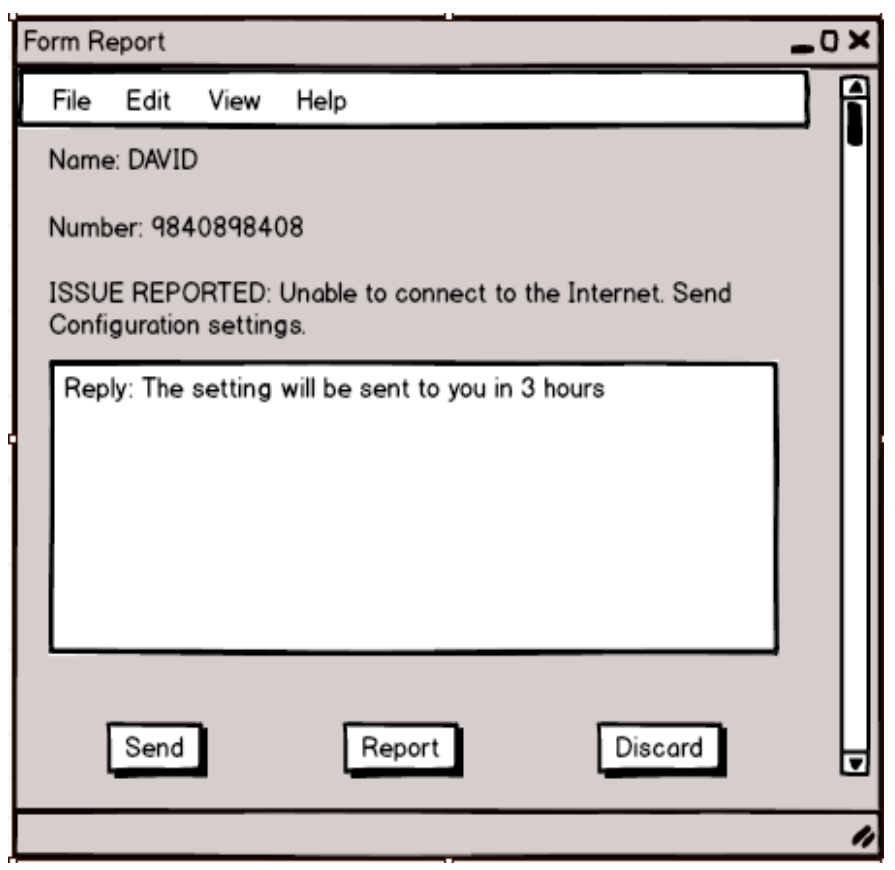

Fig. 4: Form on Web Application

The form that is received by the executive will look similar to this figure with the relevant fields. The text box is provided for the reply to be typed in and to be sent. In the case the executive finds the report as irrelevant or a nuisance one he could discard the message using the button provided.

\section{BENEFITS OF THIS SYSTEM}

i. Win-Win for both user and customer care executive.

ii. Can avoid nuisance calls.

iii. Text data transmission is cheaper that voice calls.

iv. Customer care executive's performance can be assessed for QoS and audit purpose.

v. Cost of implementation is low.

vi. Reduces man power in operations.

vii. Saves time when compared to the existing system.

viii. Dynamic storage system of data.

\section{RESULTS AND DISCUSSION}

When compared to the existing system of voice process in call centers, the usage of this system can produce a much better result in terms of time consumed.

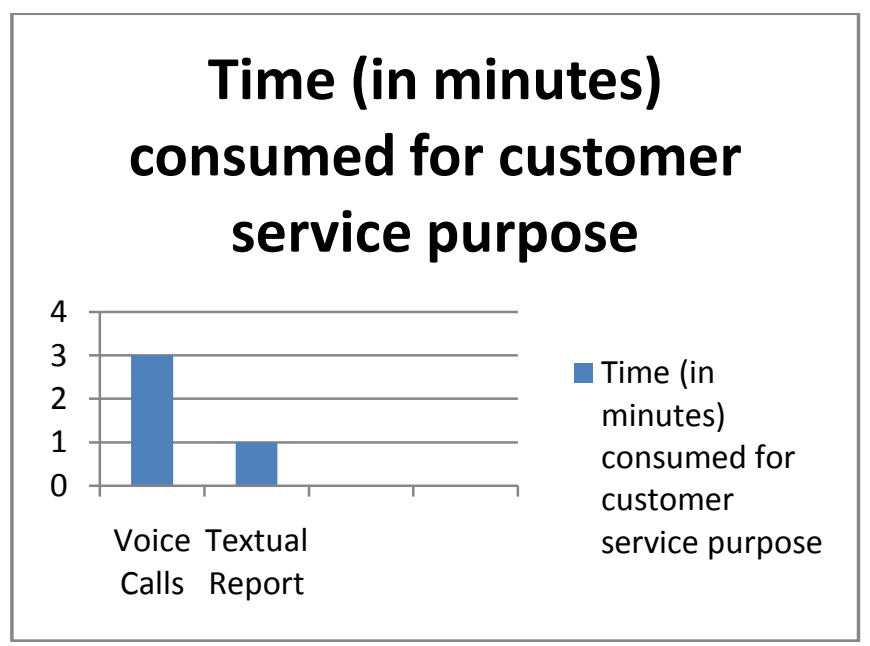

Chart 1: Graph showing Time taken for addressing

It is evident from the graph that the textual report would consume just $1 / 3$ rd of the time it would take to address an issue or provide assistance over a call.

On the other hand it is the same case with the storage of the data for review purpose later. The graph below suggests that the memory area occupied in a system to store a voice call is much higher that the memory required for storing textual data.

\section{KiloBytes required to store data}

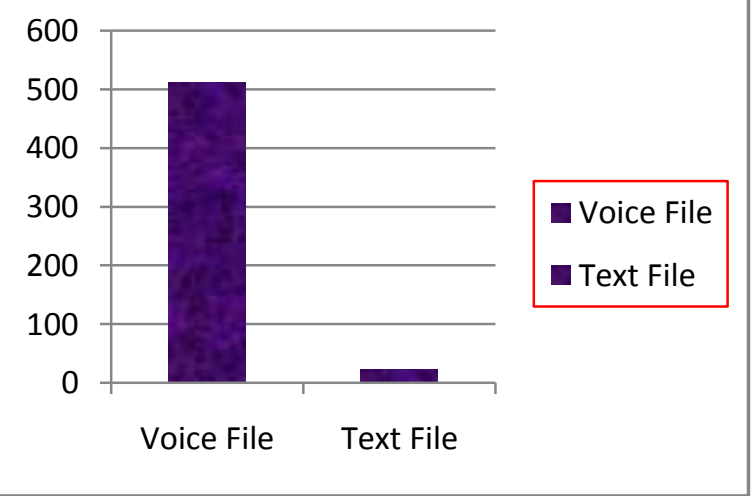

Chart 2 Graph showing memory occupied for storage 
The minimum size of a text record in a database would only be about $24 \mathrm{~KB}$ but for storing a recorded voice it will occupy a minimum of $512 \mathrm{~KB}$.

\section{CONCLUSIONS}

The proposed system when implemented will prove to be very useful and will produce excellent results in terms of both customer satisfaction as well as an economically viable option. This system will relieve the customer service executives of mundane tasks and will give then more time to handle each task. The productivity of BPO's will also be improved by its usage. In future this system can be improved based on the improvements in technology on the network side such as increase in average bandwidth. It will help perform the data transfer at a faster pace.

\section{ACKNOWLEDGEMENTS}

We the members of this project acknowledge our gratitude for the sincere support provided to us by our management for providing all the help and facilities. We also express our sincere thanks to our guide who has been an integral part in helping us throughout the entire project.

\section{REFERENCES}

[1] Design and Development of a Web Service for Android Applications for Extensive Data Processing by Robert A. Sowah, Seth Y.Fiowoo.

[2] Mobile Computing and Databases- A survey by Daniel Barbara

[3] A study of Android Application Security by Willian Enck et.al

[4] Developing Mobile Learning Applications for android Using Web service by Paul POCATILU.

\section{BIOGRAPHIES}

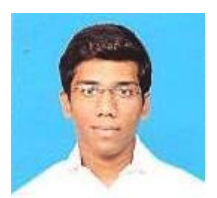

Jagadeesh Prabhakaran is a student of Panimalar Engineering College, Chennai, pursuing his bachelor's degree in computer science and engineering. He has presented and published 3 papers in international conferences. His areas of interests include mobile computing, artificial intelligence and database management system.

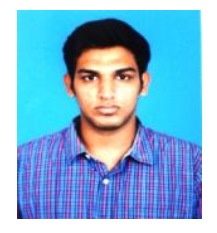

Abraham Mathulla Kandathil is also a student of Panimalar Engineering College, Chennai. He is also pursuing his bachelor's degree in computer science and engineering. His areas of interests are Object Oriented Programming and software testing.

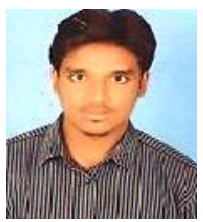

Gautham Govindharaman is also a student of Panimalar Engineering College, Chennai. $\mathrm{He}$ is also pursuing his bachelor's degree in computer science and engineering. His areas of interests are software engineering and software testing.

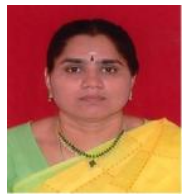

Dr. K. Valarmathi is a professor at the Department of Computer Science in Panimalar Engineering College. She has a dynamic experience of more than 15 years in the field of teaching. Her area of interest and research is computer networks. 